\title{
End Stage Dementia and Entropy Definition of Suffering
}

\author{
Bechor Z. Aminoff* \\ Geriatric Division, The Chaim Sheba Medical Center, Tel Hashomer 52621, Israel
}

\begin{abstract}
Objectives: A new definition of human suffering and satisfaction according to the entropy hypothesis may facilitate comprehension of health, disease and the aging process.

Methods: A cohort study of 71 patients (28 females, 43 males), with very advanced dementia, who died in our ward during the study period. The intense suffering level of end-stage dementia patients was evaluated by the Mini-Suffering State Examination (MSSE) scale.

Results: Suffering level in end-stage dementia has a significant correlation with short survival, advancing age, more severe illness, malnutrition, the existence of decubitus ulcers, and the administration of medications. Established correlations could be explained by the enhanced level of the patients' body entropy.
\end{abstract}

Discussion: Suffering and satisfaction are functional levels of human entropy.

An elevated level of human entropy is a measure of disorder, a process of aging and torment of the patient.

Keywords: Dementia, suffering, entropy, disease, aging.

"...the earth was unformed and void ... and God saw everything that He had made and behold, it was very good". (Genesis I; 2, 31)

\section{INTRODUCTION}

Human suffering has received limited attention in medical education, research, and practice [1-3]. Definition of suffering of healthy and sick individuals and estimation of human suffering by objective tools [4] are of profound importance in daily medical practice.

It is known that the emotional and intellectual life of human beings is composed of two principal conditions: a feeling of well-being and a condition of despair, i.e. a feeling of satisfaction and enjoyment, and a state of suffering.

Cassell $[1,5]$ believed that suffering is distress induced by the actual or perceived impending threat to the integrity or continued existence of the self as a whole. Benedict [6] defined suffering as a negative affective state resulting from an event or situation that is perceived to be physically painful, uncomfortable, or psychologically distressing.

Fishman [7] indicated that suffering is a state of mind, or a conscious experience of a person, that is created by many different influences, whereas pain is only one of these factors and its presence does not necessarily produce suffering. Portenoy [8] wrote that suffering is a global aversive experience sustained by many negative perceptions, only one of which is pain.

Chapman and Garvin [9] described suffering as a complex negative, affective and cognitive state, characterized by

*Address correspondence to this author at the Geriatric Division, The Chaim Sheba Medical Center, Tel Hashomer 52621, Israel; Tel: +972-52-6666550; Fax:+972-3-9360126; E-mail: bechorz@yahoo.com a perceived threat to the integrity of the self, perceived helplessness in the face of that threat, and exhaustion of psychosocial and personal resources of coping. The model proposed by Cherny, Coyle, Foley [10] described suffering as an aversive experience, characterized by the perception of personal distress which is generated by adverse factors that undermine quality-of-life.

Cherny [11] proposed the triangular model of suffering and taxonomy of factors, prevalence of distress experienced by patients, their families, and their attending health care professionals.

These aforementioned definitions of suffering do not adequately express the nature and origin of suffering and satisfaction, or suggest how to treat, relieve and prevent the suffering of a healthy or ailing individual.

The nature of suffering makes it difficult to assess, and there have been no reports in the medical literature on the methods of examining the level of suffering. This is in contrast to various clinical instruments that are designed to measure quality-of-life or satisfaction with care at the endof-life. Some may argue that establishing a clinical diagnosis of suffering is impossible, yet others feel that ESD represents a "persistent vegetative state" without sensation, perception, and emotion.

Moreover, the suffering of ESD patients is often accompanied by an inability to verbally indicate the extent of their suffering. We tried to approach this issue from a more objective, rather than the classic and immeasurable, subjective approach.

\section{METHODS}

We prospectively studied consecutive ESD patients, admitted to our center during a 24 -month period. Follow-up 
continued till cessation of the study. Diagnosis of dementia was made according to the DSM-4 Revised criteria [12]. Inclusion criteria comprised of severe dementia interference in verbal communication (MMSE 0/30) [13], and complete dependence in activities of daily living and functional movement (Functional Independence Measure [FIM] 18/126) [14].

We recruited patients diagnosed as suffering from Alzheimer's disease, multi-infarct dementia, post-stroke dementia, and dementia of unknown origin. The study was authorized by the local Helsinki Ethics Committee, and informed consent was given by families and caregivers.

Total number of 158 ESD patients were admitted to our ward from various intensive care departments of the hospital. Eighty-seven patients were in a stable medical condition, and most were discharged from the ward to their previous living arrangements, or to other nursing facilities. We included final analysis in the the remaining 71 patients ( 28 females, 43 males), with very advanced dementia, who died in our ward during the study period.

All patients were evaluated by the MSSE scale during the first week of admission to our geriatric department and during the last week of life. We also recorded other laboratory and clinical data relevant to the patients' condition.

\section{MINI-SUFFERING-STATE-EXAMINATION (MSSE) SCALE}

However, it is most unlikely that the association between subjective suffering and objective clinical tools is identical for end-of-life dementia patients, and for those with a less advanced stage of disease. We strongly believe that despite of severe cognitive impairment, sensation and emotional status of these patients are only partially impaired, in which case probability of suffering is considerable. Objective, wellvalidated tools should be used to evaluate the suffering level.

Our recently developed Mini-Suffering-State-Examination (MSSE) scale is the first objective clinical tool for the evaluation of suffering level in ESD [4].

The MSSE scale comprises 10 items, related to the patients' characteristics, as well as the perception of their condition by medical staff and families. Each item scores 0 (no) or 1 (yes). Total score ranges between 0-10; high scores reflect higher degrees of suffering. Based on clinical experience, the following items were included in the MSSE: Not calm, screams, pain, decubitus ulcers, malnutrition, eating disorders, invasive action, unstable medical condition, suffering according to medical opinion, and suffering according to family opinion.

The MSSE is brief and it takes less than 10 minutes of the physician or nurse to evaluate the suffering level of the patient. The significant reliability of the MSSE scale was demonstrated by using the Cronbach- $\alpha$ model $(0.798)$. Concurrent validity of the MSSE scale was proved by Pearson correlation with the Symptom Management with End-of-Life in Dementia (SM-EOLD) scale $(\mathrm{r}=0.574, P<0.0001)$, and the Comfort Assessment in Dying with Dementia (CADEOLD) scale [15] $(\mathrm{r}=-0.796, P<0.0001)$.

\section{STATISTICAL ANALYSIS}

Comparisons between the three levels of MSSE (low, intermediate, and high) with regard to demographic and clinical variables, were performed using analysis of variance (ANOVA), Kruskal Wallis non-parametric test, and Fisher's exact test, where applicable. The statistical significance level was set to 0.05 , and the SPSS for Windows software, version 11.0 , was used for the analysis.

\section{RESULTS}

Eighty-seven patients, who were in a stable medical condition and most were discharged from the ward, were in low suffering level and had MSSE score $=3.61 \pm 2.0$

The results of our research showed that care in the geriatric department failed to reduce the high level of suffering of dying ESD patients. The suffering level of an ESD patient increased until demise (Table 1). Our research concluded that $63 \%$ and $30 \%$ of dementia patients died with a high and intermediate level of suffering, respectively. Only $7 \%$ died with a low level of suffering. On the day of admission, the total score of the MSSE scale was $5.62 \pm 2.31$, and increased to $6.89 \pm 1.95$ on the last day of life, with a significant difference $(P<0.0001)$.

Table 1. Correlation Between Mini Suffering State Examination Scale Score and Survival Time in End Stage Dementia

\begin{tabular}{|c|c|c|c|c|}
\hline Category & $\mathbf{N}$ & $\mathbf{\%}$ & MSSE Score & Survival Time (Days) \\
\hline \hline Low & 5 & 7 & $2.24 \pm 0.99$ & $57.76 \pm 9.73$ \\
\hline Medium & 21 & 30 & $4.92 \pm 0.83$ & $44.70 \pm 5.99$ \\
\hline High & 45 & 63 & $8.06 \pm 1.00$ & $27.54 \pm 4.16$ \\
\hline
\end{tabular}

Mean survival time was $57.76 \pm 9.73$ days for the low MSSE score group (MSSE $=2.24 \pm 0.99), 44.70 \pm 5.99$ days for the median MSSE score group (MSSE $=4.92 \pm 0.83$ ), and $27.54 \pm 4.16$ days for the high MSSE score group (MSSE $=8.06 \pm 1.00$ ). Differences between the survival times of these three MSSE score groups were statistically significant (Kaplan-Meier Analysis Log Rank Test, $P=0.0018$; Breslow Test, $P=0.0027$ ).

The Cox proportional Hazard model of survival revealed a significant interrelation of high MSSE scores and shorter survival $(P=0.013)$.

\section{DISCUSSION}

Results of our clinical study demonstrated that the intense suffering level of patients evaluated by a recently developed objective tool, the Mini-Suffering State Examination (MSSE) scale, has a significant correlation with short survival [16], advancing age, more severe illness, malnutrition, the existence of decubitus ulcers, and the administration of medications [17].

It was also found that the suffering level of end-stage dementia (ESD) patients $[18,19]$ and the struggle of family 
members [20] escalated during the dying process, until demise. Established correlations could hypothetically be explained by the enhanced level of the patients' body entropy.

Entropy may be defined as a measure of chaos, or a measure of lack of order. If the system is perfect, managed correctly or any other description that can define the state of order, then the entropy of the system is lowered accordingly, and is thus defined as a process of the depression of entropy, negative entropy or anti-entropy.

The incentive to attain positive emotions is the mechanism of progress and creativity in science, art, economics, sport or other disciplines.

From the moment a baby first smiles, and all subsequent achievements and progress, whether physical or intellectual throughout life, whether related to family or a career, contribute in diminishing chaos and entropy. Thus, foundations are created for satisfaction, happiness, pleasure, joy, pride, and other positive emotions of well-being. The constant motivation towards progress, advancement, improvement and acquisition, may reduce the level of entropy and generate a feeling of pleasure and, thus, well-being.

What then is suffering and satisfaction? First, these are sensations. We know that humans possess five senses: hear- ing, sight, smell, taste, and touch. An odor may be repugnant or fragrant. Food may be appetizing or bland. A touch may be pleasant, such as that of a baby's silky skin, or agonizing, such as the touch of burning metal. The sight of flowers blossoming in a field produces a sense of joy, but we are devastated by the destruction of war. We enjoy listening good music, and are distressed by cacophony.

Suffering and satisfaction are also positive perceptions that can cause either suffering or pleasure. The main perception of suffering is commonly referred to as pain. The important ingredient of suffering and satisfaction is emotion.

Positive emotions are mentioned in the seven blessings: joy and gladness, groom and bride, mirth and song, delight, love, brotherhood, peace, and companionship. Negative emotions, such as fear, anxiety, tension, anger, frustration, concern, helplessness, and depression, are all sources of suffering. Cheerful and distressing thoughts are sources of either enjoyment or suffering and are also prevalent in cognitive life.

Our hypothesis is that suffering and satisfaction are functions of the level of human entropy [21]. Fig. (1) shows, the possible correlation between the hypothetical levels of human suffering and entropy from the moment of birth until

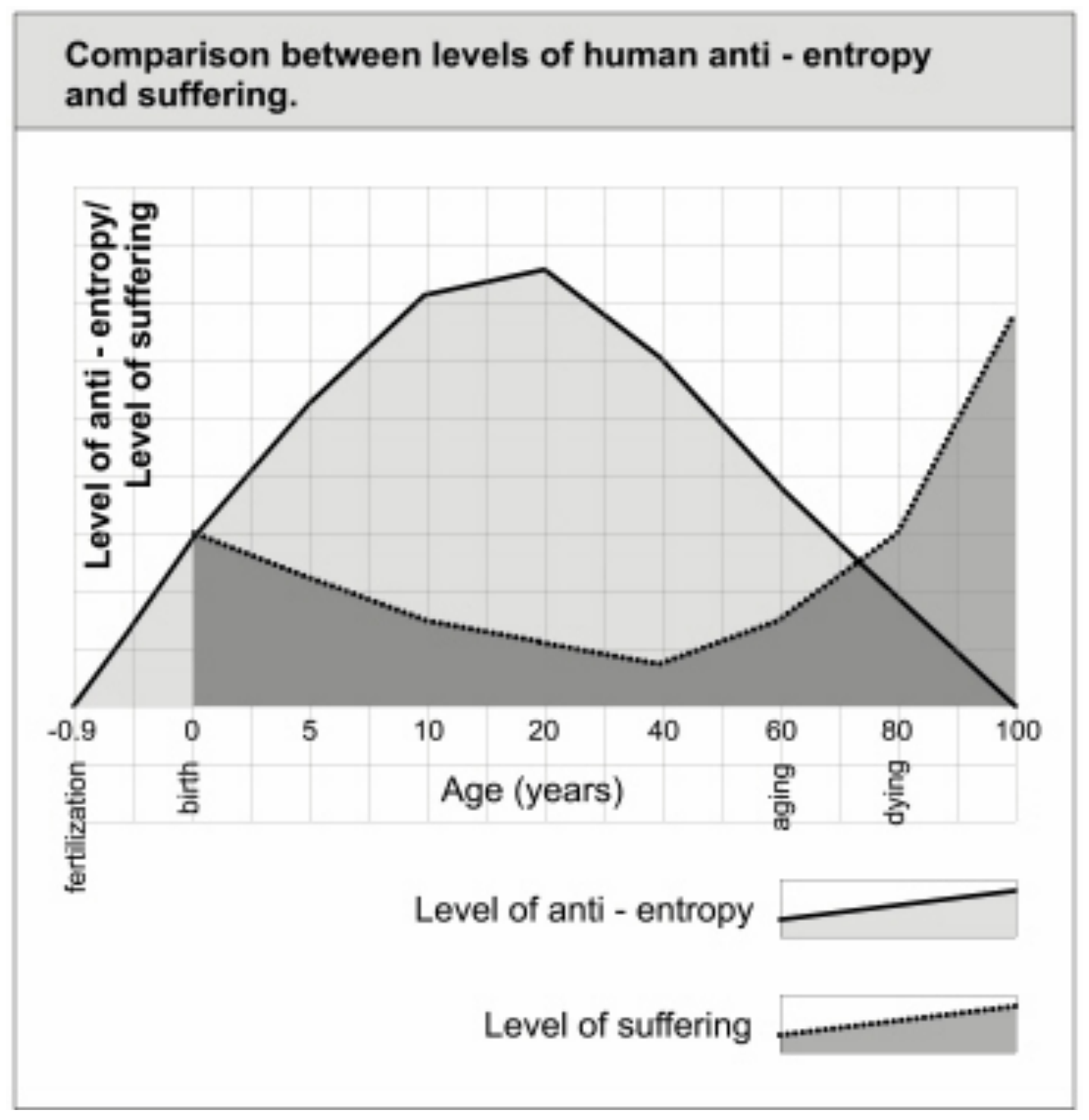

Fig. (1). Comparison between levels of human anti-entropy and suffering. 
demise of the individual, which should be investigated and proved in future clinical research.

We believed that human suffering may be defined as a complexity of negative sensations, perceptions, emotions or human thoughts that arise due to an increasing level of entropy of a person's organism and are affected by relationships with others, or germane surroundings in the past, present, or a future threat.

On the other hand, human satisfaction may be defined as the complexity of positive sensations, perceptions, emotions or thoughts of a person that arise due to a depressive level of entropy of an individual's organism and empathy for others, or relevant surroundings in the past, present or even the future.

Every loss, disruption, or deterioration in organization and lack of order implied an increase in the entropy level, and is, therefore, a source of suffering. Thus, the aging process intensifies the level of entropy; a process of progressive loss of reserves of the system until survival is impossible, and the individual passes away [22].

Diseases can also be a natural mishap in the structure and organization of the body $[23,24]$. All these processes cause a rise in the entropy level and are sources of suffering. Not only a human being as an individual unit being discussed, but rather a human being as a part of the system. Subsequently, a rise in the entropy level of a link in the system generates a rise in the entropy of the whole system, leading to suffering.

Degenerative brain diseases, such as Alzheimer's and Parkinson's, are caused by an increased level of entropy and deterioration of the brain $[25,26]$ that forms the basic concept of an individual. Disruptions and damage to brain functions, particularly at the last stage of illness, when all attempts of recovery are futile, result in an increased level of entropy and are a source of immense suffering to the sick patient $[17,18]$, to the family, and to all those who are closely associated.

Thus, in order to treat suffering, one should ensure that the level of entropy is lowered, by replacing the deficiency that causes disturbances. As the level of entropy and suffering increases, care and treatment should be adjusted accordingly [27].

\section{REFERENCES}

[1] Cassell EJ. The nature of suffering and the goals of medicine. N Engl J Med 1982; 306: 639-45.

[2] Lesho EP. When the spirit hurts. Arch Intern Med 2003; 163: 242932.

[3] Aminoff BZ. Measurement of suffering in end-stage Alzheimer's disease. $1^{\text {st }}$ ed. Tel-Aviv:Probook, Dyonon 2007.
[4] Aminoff BZ, Purits E, Noy S, Adunsky A. Measuring the suffering in end-stage dementia: Reliability and validity of the Mini Suffering State Examination. Arch Gerontol Geriatr 2004; 38: 123-30.

[5] Cassell EJ. The importance of understanding suffering for clinical ethics. Clin Ethics 1991; 2: 81-2.

[6] Benedict S. The suffering associated with lung cancer. Cancer Nurse 1989; 12: 34-40.

[7] Fishman B. The treatment of suffering in patients with cancer pain. In: Foley KM, Bonica JJ, Ventafridda V, Eds. Advances in pain research and therapy, New York: Raven Press 1990; vol. 16: pp. 301316.

[8] Portenoy RK. Pain and quality of life: Clinical issues and implications for research. Oncology 1990; 4: 172-8.

[9] Chapman CR, Garvin J. Suffering and its relationship to pain. J Palliat Care 1993; 9: 5-13.

[10] Cherny NI, Coyle N, Foley KM. Suffering in the advanced cancer patient: A definition and taxonomy. J Palliat Care 1994; 10: 57-70.

[11] Cherny NI. The challenge of palliative medicine. The problem of suffering. In: Doyle D, Hanks G, Cherny NI, Calman K, Eds. Oxford textbook of palliative medicine, $3^{\text {rd }}$ ed. Oxford: Oxford University Press 2004; pp. 7-14.

[12] American Psychiatric Association. Diagnostic and Statistical Manual of Mental Disorders, $4^{\text {th }}$ ed: Washington DC 1994.

[13] Folstein MF, Folstein SE, McHugh PR. "Mini-mental state". A practical method for grading the cognitive state of patients for the clinician. J Psychiatr Res 1975; 12: 189-98.

[14] Granger CV, Hamilton BB. The uniform data system for medical rehabilitation: Report of first admissions for 1992. Am J Phys Med Rehabil 1992; 73: 51-5.

[15] Volicer L, Hurley CA, Blasi VZ. Scales for evaluation of end-oflife care in dementia. Alzheimer Dis Assoc Disord 2001; 15: 194200.

[16] Aminoff BZ. Mini-Suffering State Examination Scale: possible key criterion for 6 months' survival and mortality of critically ill dementia patients. Am J Hosp Palliat Med 2008; 24: 470-4.

[17] Aminoff BZ, Adunsky A. Dying dementia patients: too much suffering, too little palliation. Am J Alzheimer Dis Others Demen 2004; $19: 243-7$.

[18] Aminoff BZ, Adunsky A. Dying dementia patients: Too much suffering, too little palliation. Am J Hosp Palliat Med 2005; 22: 344-8.

[19] Aminoff BZ, Adunsky A. Their last six months: Suffering and survival of end stage dementia patients. Age Ageing 2006; 35: 597601.

[20] Aminoff BZ. Overprotection phenomenon of dying dementia patients. (Editorial). Am J Hosp Palliat Med 2005; 22: 247-8.

[21] Aminoff BZ. Suffering, satisfaction and entropy. (Abstract). $17^{\text {th }}$ World Congress of the International Association of Gerontology, Vancouver, Canada. Gerontology (Abstract) 2001; 47: 124.

[22] Allen PA, Kaufman M, Smith AF, Propper AE. A molar entropy model of age differences in spatial memory. Psychol Aging 1998; 13: 501-18.

[23] Cohen PG. Aromatase, adiposity, aging and disease. The hypogonadal-metabolic-atherogenic-disease and aging connection. Med Hypotheses 2001; 56: 702-8.

[24] Pezard L, Martinerie J, Varela FJ, et al. Entropy maps characterize drug effects on brain dynamics in Alzheimer disease. Neurosci Lett 1998; 253: 5-8.

[25] Riggs JE. Aging, increasing genomic entropy, and deurodegenerative disease. Neurol Clin 1998; 16: 757-70.

[26] Drachman AD. Aging of the brain, entropy, and Alzheimer disease. Neurology 2006; 67: 1340-52.

[27] Aminoff BZ. End-stage dementia: Aminoff suffering syndrome and relief of suffering units. Open Geriatr Med J 2008; 1: 29-32. 